\title{
Delay Guaranteed Fair Queueing(DGFQ) in Multimedia Packet Networks
}

\author{
Hyunho Yang ${ }^{1}$ and Kiseon $\mathrm{Kim}^{2}$ \\ 1 Dept. of Computer and Information, Suncheon Cheongam College, \\ 224-9, Deogweol-dong, Suncheon-si, Jeonnam, 540-743, Republic of Korea. \\ hhyang@scjc.ac.kr \\ 2 Dept. of Information and Communications, \\ Kwangju Institute of Science and Technology(K-JIST) \\ 1 Oryong-dong, Puk-gu, Kwangju, 500-712, Republic of Korea \\ kskim@kjist.ac.kr
}

\begin{abstract}
Fair queueing has been an important issue in the multimedia networks where resources are shared among nodes both wired and wireless. Most fair queuing algorithms based on the generalized processor sharing (GPS) emphasize fairness and bounded delay guarantee, while overlooking service differentiation for different service classes of their own delay bounds. In this paper, we propose a new fair queueing scheme, delay guaranteed fair queueing (DGFQ), which guarantees bounded delay for the classes of flows according to their individual delay requirements for multimedia services in the wireless packet networks.
\end{abstract}

Keywords. Fair Queueing, Bounded Delay, Quality of Service (QoS), Multimedia Network.

\section{Introduction}

The telecommunication technologies are envisioned to support multimedia services, both error-sensitive and delay-sensitive applications, even over the bandwidth-constrained wireless medium. With this vision in mind, the issue of providing fair and bounded delay channel access among multiple contending hosts over a shared channel resources has come to the fore. To achieve this goal, fair queueing has been a popular paradigm in both wireline and wireless packet cellular networking environments [1].

A series of algorithms are based on generalized processor sharing (GPS) 22 approach. Among them are WFQ 3] also called pack-by-packet GPS (PGPS), self clocked fair queueing (SCFQ) [4, and start time fair queueing (SFQ) [5. These algorithms guarantee the fairness according to the relative weight of flows. Many other wireless fair queueing algorithms have been proposed as a variation of these algorithms.

In [5], the authors reviewed typical GPS based fair queueing algorithms and proposed a new simple, deadline guaranteeing fair queueing algorithm, i.e., starttime fair queueing (SFQ). Their proposed scheme implemented virtual time function less complex by adopting start tag of currently transmitting packet as virtual 
time value. In addition, SFQ allocates bandwidth fairly regardless of admission control as well as variation in server rate. Though this scheme improves delay guarantee and fairness bound, the authors overlooked the service differentiation according to the flow characteristics, e.g., guaranteed bandwidth service or best effort service, which is critical to support multiple heterogeneous sessions traffics both realtime and non realtime.

The delay and data decoupled fair queueing (D-FQ) was presented in [7, to mitigate the inherent drawback of GPS based fair queueing algorithm i.e., the inability to deliver independent control over delay and bandwidth guarantees, which results in inflexible bandwidth management and inefficient link utilization 8. Their approach for independent control for delay and data rate is to sperate scheduling algorithms for real time and non-real time flows. This scheme not only guarantees the delay deadline of real time flows but also improves the performance of non-real time flows in terms of packet delay, however, it should be pointed out that the computational complexity increases due to the dual scheduling architecture, specifically for the explicit-delay-guarantee(EDG) algorithm which support real time flows.

In this paper, we propose a new fair queueing scheme i.e., delay guaranteed fair queueing (DGFQ), guaranteeing bounded delay of multimedia services. Our model is based on GPS algorithm with some modifications to guarantee bounded delay. In detail, we categorized the flows into two classes, i.e., delay guaranteed (DG) and non-delay guaranteed (NG). Then introduce additional weight factor to apply differentiated tagging operation for each class, i.e., set a little earlier start tag to DG class flows than to NG class flows. With this policy we can get better delay performance for DG class flows at the same fairness guarantee without serious increase in computational complexity.

The rest of this paper is organized as follows. Section 2 is devoted to describe the network model being considered. In Section [3, detailed architecture and algorithm of delay guaranteed fair queueing (DFGQ) is given. Then in Section 4 the performance of proposed scheme is evaluated. Finally, we concluded our work in Section 5

\section{Network Model}

For the purpose of this work, we basically consider wireless networks supporting multimedia services with guaranteed delay performance. We also expect that this scheme performs similarly for the wired network.

In detail, we consider packet-cellular network with a high-speed wired backbone and small, partially overlapping, shared channel wireless cells. Each cell is served by a base station, which performs the scheduling of packet transmissions for the cell. Neighboring cells are assumed to transmit on different logical channels. Every mobile host in a cell can communicate with the base station, though it is not required for any two mobile hosts to be within range of each other. Each flow of packets are identified by a certain flow index. To concentrate our work on the enhancement of the delay guarantee performance, we excluded channel 
error to leave as a future work. Thus, we do not make any explicit mathematical assumptions about the error model in our framework. It should be noted that in our model, we do not consider the scenario where a wireless channel is shared across several neighboring cells, which is more complicated and introduces the hidden/exposed station problems [9].

\section{Delay Guaranteed Fair Queueing(DGFQ)}

In this section, we propose a new fair queueing algorithm, delay guaranteed fair queueing (DGFQ), which provides differentiated bounded delay for each traffic class having different service requirements. This proposal includes algorithm description, analytical properties and implementation in error free channel.

\subsection{Algorithm Descriptions}

Our proposed model, delay guaranteed fair queueing (DGFQ) basically adopts start-time fair queueing (SFQ) algorithm proposed in [5]. In DGFQ, as is in the SFQ, two tags i.e., a start tag and a finish tag, are associated with each packet. However, unlike WFQ and SCFQ, packets are scheduled in the increasing order of the start tags of the packets. Furthermore, $v(t)$ is defined as the start tag of the packet in service at time $t$. Finally, we assume that, in SFQ, WFQ or DGFQ scheme, there is a certain interval of time in which all flows are scheduled at least once, we call it scheduling interval.

It is desirable to categorize flows into classes to provide QoS for multimedia services, specifically for IP networks. 6]. To adopt this policy, all flows are classified into a number of classes according to their delay bound requirements. The simplest and basic classification is to make two classes, one for delay guaranteed (DG) flows and the rest for non delay guaranteed (NG) flows.

In all GPS based queueing algorithms, the weight parameter $\phi_{f}$ for a flow $f$ is defined as

$$
\phi_{f}=\frac{r_{f}}{\sum_{j \in B} r_{j}}
$$

where $B$ is a set of backlogged flows. It is intuitively evident from the above definition that $\phi_{f}$ is strongly dependent on the relative rate of a flow to the total backlogged flows in the system at a certain time instant. Thus, an additional weight parameter is required to control delay performance, hence, QoS, for each group of flows.

In our scheme, we introduce the service differentiation coefficient, $\alpha(0<$ $\alpha \leq 1$ ), to handle each flow class differently. When $\alpha=1$, which is the case for NG class, our proposed scheme is identical to SFQ. By varying $\alpha$, we can customize delay bound for individual flows i.e., adjust the relative service order of each flow in a scheduling interval.

The complete algorithm is defined as follows. 


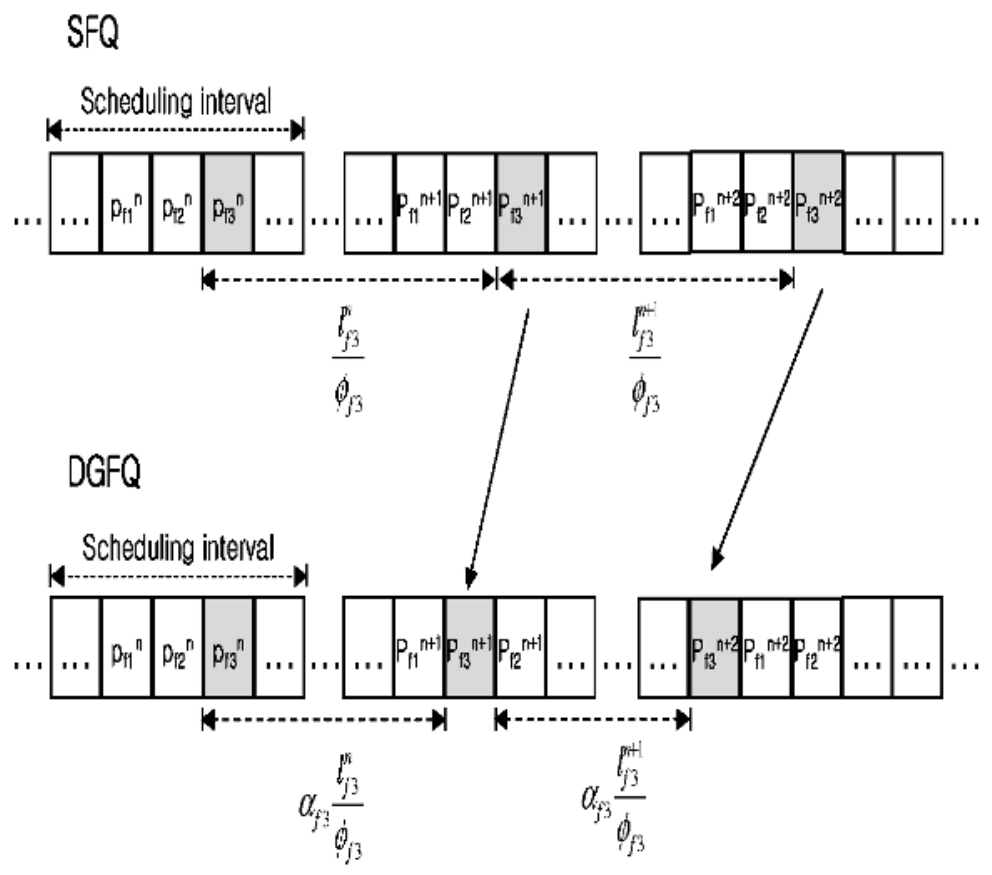

Fig. 1. Comparison of Packet Scheduling Schemes (SFQ v.s DGFQ)

1. On arrival, $k^{t h}$ packet of flow $f, p_{f}^{k}$, is stamped with start tag $S\left(p_{f}^{k}\right)$, computed as

$$
S\left(p_{f}^{k}\right)=\max \left\{v\left[A\left(p_{f}^{k}\right)\right], F\left(p_{f}^{k-1}\right)\right\} \quad k \geq 1
$$

where $A\left(p_{f}^{k}\right)$ is the arrival time of packet $p_{f}^{k}$ and $F\left(p_{f}^{k}\right)$ is the finish tag of packet $p_{f}^{k}$, defined as

$$
F\left(p_{f}^{k}\right)=S\left(p_{f}^{k}\right)+\alpha_{f} \frac{l_{f}^{k}}{\phi_{f}}
$$

where $F\left(p_{f}^{0}\right)=0, \phi_{f}$ is the weight of flow $f, l_{f}^{k}$ is the length of packet $p_{f}^{k}$, and $\alpha\left(0<\alpha_{f} \leq 1\right)$ is the service differentiation coefficient for flow $f$. The value of $\alpha$ is 1 for NG class, or appropriate value for DG class.

2. Initially the system virtual time is 0 . During a busy period, the system virtual time at time $t, v(t)$, is defined to be equal to the start tag of the packet in service at time $t$. At the end of a busy period, $v(t)$ is set to the maximum of finish tag assigned to any packets that have been serviced by then.

3. Packets are serviced in the increasing order of the start tags, ties are broken arbitrarily. 
As shown in the Fig. 回 in DGFQ, the next packet is scheduled after $\alpha_{f} \frac{l_{f}^{k}}{\phi_{f}}$, while in SFQ scheme, the next packets of each on going flow scheduled after $\frac{l_{f}^{k}}{\phi_{f}}$. As mentioned before, the range of $\alpha$ is $0<\alpha \leq 1$, the scheduling time difference i.e., $\left(1-\alpha_{f}\right) \frac{l_{f}^{k}}{\phi_{f}}$, is limited to one packet transmission duration. It is evident from (11) and (2) that the differentiated service time (virtual start time of a packet) can not go before system virtual time of the packet arrival instant. Due to this property, DGFQ maintain the fairness between each flow. On the other hand, it is still another issue to compensate lagged service in the error-pron channel environment, thus exclude from our considerations. Finally, the computational complexity of computing virtual time $v(t)$ in DGFQ is $O(\log N)$ per packet where $N$ is the number of flows in the system.

\subsection{Analytical Properties of DGFQ}

In this section, the analytical properties of DGFQ are discussed specifically for the fairness guarantee and delay guarantee.

Fairness Guarantee. To derive fairness guarantee of DGFQ, let $W_{f}\left(t_{1}, t_{2}\right)$ and $W_{g}\left(t_{1}, t_{2}\right)$ be the amount of service received by flows $f$ and $g$ in the time interval $\left[t_{1}, t_{2}\right]$, moreover, $\phi_{f}$ and $\phi_{g}$ be the weight of each flow respectively. We need to prove a bound on

$$
\left|\frac{W_{f}\left(t_{1}, t_{2}\right)}{\phi_{f}}-\frac{W_{g}\left(t_{1}, t_{2}\right)}{\phi_{g}}\right|
$$

for any interval in which both flows $f$ and $g$ are backlogged. We achieve this objective by establishing a lower and an upper bound on $W_{f}\left(t_{1}, t_{2}\right)$ and $W_{g}\left(t_{1}, t_{2}\right)$ in Lemmas 1 and 2 respectively.

Lemma 1. If flow $f$ is backlogged throughout the interval $\left[t_{1}, t_{2}\right]$, then in an $D G F Q$ server

$$
\phi_{f}\left(v_{2}-v_{1}\right)-l_{f}^{\max } \leq W_{f}\left(t_{1}, t_{2}\right)
$$

where $v_{1}=v\left(t_{1}\right)$ and $v_{2}=v\left(t_{2}\right)$ are virtual time of each time instant $t_{1}, t_{2}$.

Lemma 2. In an $D G F Q$ server, during any interval $\left[t_{1}, t_{2}\right]$

$$
\left.W_{g} t_{1}, t_{2}\right) \leq \phi_{g}\left(v_{2}-v_{1}\right)+l_{g}^{\max }
$$

where $v_{1}=v\left(t_{1}\right)$ and $v_{2}=v\left(t_{2}\right)$ are virtual time of each time instant $t_{1}, t_{2}$.

Since unfairness between two flows in any interval is maximized when one flow receives maximum possible service and the other minimum service, Theorem 廿10 follows directly from Lemmas 1 and 2 
Theorem 1. For any interval $\left[t_{1}, t_{2}\right]$ in which flows $f$ and $g$ are backlogged during the entire interval, the difference in the service received by two flows at an DGFQ server is given as

$$
\left|\frac{W_{f}\left(t_{1}, t_{2}\right)}{\phi_{f}}-\frac{W_{g}\left(t_{1}, t_{2}\right)}{\phi_{g}}\right| \leq \frac{l_{f}^{\max }}{\phi_{f}}+\frac{l_{g}^{\max }}{\phi_{g}}
$$

Theorem 1 demonstrates that DGFQ guarantees the same fairness as in SFQ [5. This is intuitively evident that DGFQ only adjusts the start tag, hence the scheduling order, in a set of backlogged flows to be serviced in a given time interval $\left[t_{1}, t_{2}\right]$, to insure earlier service for delay guaranteed flows.

Delay Guarantee. On the contrary to the fairness guarantee, DGFQ shows better performance than SFQ, for the delay guaranteed (DG) flows such as real time videos. Let $r_{f}$ be the rate assigned to packet $p_{f}^{k}$ length of $l_{f}^{k}$. Then finish tag of packet $p_{f}^{k}, F\left(p_{f}^{k}\right)$ is defined as

$$
F\left(p_{f}^{k}\right)=S\left(p_{f}^{k}\right)+\alpha_{f} \frac{l_{f}^{k}}{r_{f}^{k}} \quad k \geq 1
$$

Start tag of a packet and the system virtual time are defined as before.

As in SFQ, DGFQ provides deadline guarantees when the server capacity is not exceeded. To drive the deadline guarantee, let us formalize the meaning of the term "capacity is not exceed." Let rate function for flow $f$ at virtual time $v$, denoted by $R_{f}(v)$, be defined as the rate assigned to the packet that has start tag less than $v$ and finish tag greater than $v$. Formally

$$
R_{f}(v)=\left\{\begin{array}{lr}
r_{f}^{k} & \text { if } \exists k \ni\left[S\left(p_{f}^{k}\right) \leq v<F\left(p_{f}^{k}\right)\right] \\
0 & \text { otherwise. }
\end{array}\right.
$$

Let $B$ be the set of flows served by the server. Then the capacity of a server with average rate $C$ is not exceeded if

$$
\sum_{n \in B} R_{n}(v) \leq C \quad v \geq 0
$$

To derive the delay guarantee of DGFQ servers, we first derive a bound on the work done by an DGFQ server in the virtual time interval $\left[v_{1}, v_{2}\right]$ in Lemma 3

Lemma 3. If the server with parameter $C$ is not exceeded, then the aggregate length of packets that have start tag at least $v_{1}$ and at most $v_{2}$, and are served in the same busy period, denoted by $\hat{W}\left(v_{1}, v_{2}\right)$, is given by

$$
\hat{W}\left(v_{1}, v_{2}\right) \leq C \sum_{n=0}^{n=k-m-1} \frac{l_{f}^{m+n}}{r_{f}^{m+n}}+\sum_{n \in B \wedge n \neq f} l_{n}^{\max }+l_{f}^{k} .
$$

whenever 


$$
v_{1}=S\left(p_{f}^{m}, r_{f}^{m}\right), \quad v_{2}=S\left(p_{f}^{k}, r_{f}^{k}\right)
$$

and

$$
v_{2}-v_{1}=\sum_{n=0}^{n=k-m-1} \frac{l_{f}^{m+n}}{r_{f}^{m+n}} .
$$

where $S\left(p_{f}^{k}, r_{f}^{k}\right)$ is the start tag of packet $p_{f}^{k}$ of flow $f$ with rate $r_{f}^{k}$.

For brevity, we will denote

$$
\theta_{f}^{k}=\sum_{n \in B \wedge n \neq f} \frac{l_{n}^{\max }}{C}+\frac{l_{f}^{k}}{C}
$$

Further, we can define $A_{e}\left(p_{f}^{k}, r_{f}^{k}\right)$, the expected arrival time of packet $p_{f}^{k}$ that has been assigned rate $r_{f}^{k}$ as

$$
A_{e}\left(p_{f}^{k}, r_{f}^{k}\right)=\max \left\{A\left(p_{f}^{k}\right), A_{e}\left(p_{f}^{k-1}, r_{f}^{k-1}\right)+\alpha_{f} \frac{l_{f}^{k-1}}{r_{f}^{k-1}}\right\}
$$

where $A_{e}\left(p_{f}^{0}, r_{f}^{0}\right)=-\infty$.

Theorem 2 defines the delay guarantee of DGFQ servers.

Theorem 2. If the capacity of DGFQ server with parameter $C$ is not exceed, then the departure time of packet $p_{f}^{j}, D_{D G F Q}$ satisfies the following inequality.

$$
D_{D G F Q}\left(p_{f}^{k}\right) \leq A_{e}\left(p_{f}^{k}, r_{f}^{k}\right)+\theta_{f}^{k}
$$

To compare this with SFQ, we can rewrite (10) as follows

$$
D_{D G F Q}\left(p_{f}^{k}\right) \leq A_{e}\left(p_{f}^{k-1}, r_{f}^{k-1}\right)+\alpha_{f} \frac{l_{f}^{k-1}}{r_{f}^{k-1}}+\theta_{f}^{k}
$$

and the departure time of a packet at a SFQ server, given in [5] is

$$
D_{S F Q}\left(p_{f}^{k}\right) \leq A_{e}\left(p_{f}^{k-1}, r_{f}^{k-1}\right)+\frac{l_{f}^{k-1}}{r_{f}^{k-1}}+\theta_{f}^{k}
$$

The difference of (11) and (12) is

$$
\left(1-\alpha_{f}\right) \frac{l_{f}^{k-1}}{r_{f}^{k-1}}
$$

where $0<\alpha_{f} \leq 1$. It is clear from the comparison above that, in DGFQ, the departure time of packet $p_{f}^{k}$ is earlier by (13) than in SFQ. Hence, the delay bound is more tightly provided than in SFQ. 


\subsection{Implementation of DGFQ in Error-Free Channel}

In this section, we consider about the implementation of the propose algorithm, delay guaranteed fair queueing (DFGQ). In the implementation, both real time and non-real time flows are scheduled based on the proposed packetized DGFQ algorithm.

To concentrate our work on the enhancement of the delay guarantee performance, we excluded channel error. It is still another issue to compensate lagged service in the error-pron channel environment. Thus, we do not make any explicit mathematical assumptions about the error model in our framework.

We assume that a transmission link is composed of time slots with fixed duration. Let $L$ and $l_{i}$ be a slot size and the packet size of a flow $i$ in bits, respectively. Let us again assume $l_{i}=n L$ for a flow $i$, where $n$ is a positive integer. Without loss of generality we assume that a time instant $t$ indicates a slot which lasts during $[t, t+\tau)$, where $\tau$ is a slot duration.

Whenever packets (single or burst of packets) from on going flows arrive, one or more time slot are assigned in the service order table. The service order table has the following properties:

- it is a queue architecture handling packets from each flow

- its elements contain the following information; flow type, flow index, service tag which is the reserved service time instant for a packet

- its elements are sorted in ascending service tag order and handled as in a single linked list.

Each arrived packet is inserted into the service order table and serviced one by one from the head of table at the start of time slots. Even though the packets are served at the beginning of each time slot, the virtual start time calculated by (11) and (2) is not quantized to a discrete time instant, i.e., the starting points of slots. It is because the virtual service time tag is used as the index key to sort the relative order in the service order table and due to this policy, each packet could be differentiated in service order during a given scheduling period.

\section{Performance Evaluation}

In this section we evaluate the performance of DGFQ, in the measurement indices i.e., throughput, average delay and maximum delay. First we described the simulation environment then, discussed about the results.

\subsection{Simulation Environments}

We used simulations method to evaluate our proposed algorithm. For the simulation, we assumed 2Mbps wireless channel which is typical capacity of current wireless mobile networks. We implemented DGFQ scheme in error free channel model to concentrate our evaluation work on the key features of proposed scheme, i.e., delay guaranteed scheduling. In the simulation, we selected average 
Table 1. Summary of Traffic Source.

\begin{tabular}{|l||c|c|c|}
\hline & Voice & Video & Data \\
\hline \hline Model & $\begin{array}{c}\text { ON/OFF } \\
(\text { ON:OFF }=1: 1.35)\end{array}$ & $\begin{array}{c}\text { Modified } \\
\text { MPEG }\end{array}$ & $\begin{array}{c}\text { Poission \& } \\
\text { Truncated Pareto }\end{array}$ \\
\hline Delay Deadline & $20 \mathrm{~ms}$ & $1000 / 24 \mathrm{~ms}$ & - \\
\hline Data Rate & $32 \mathrm{kbps}$ & $114 \mathrm{kbps}$ & $300 \mathrm{kbps}$ \\
\hline Relative Weight & 0.0016 & 0.057 & 0.15 \\
\hline
\end{tabular}

delay, maximum delay and throughput as the performance measures. Detail definitions of these measure are explained in the following Section 4.3 Moreover, we compared these measures for the following three fair queueing schemes; SFQ, DGFQ, D-FQ 7. SFQ also regarded as a special case of DFGQ where the service differentiation coefficient $\alpha=1.0$. For the case of D-FQ is simulated to get the reference value of the delay performance because it is explicitly guarantee delay deadline with somewhat costly complex system architecture with computational complexity of $O\left(N_{A W} N_{R T}\right)$ where $N_{A W}$ is the number of allocation windows and $N_{R T}$ is the number of realtime flows [7, and it also meaningful to compare the performance of our simple scheme with computational complexity of $O(\log N)$ where $N$ is the number of flows in the system. Simulation performed for 1000 seconds, further, repeated 20 times to get more refined results.

\subsection{Traffic Source Models}

In this work we choose the same traffic model used in 7 to make the results be comparable. In the simulation, a voice flow is modelled as ON-OFF signal with $\mathrm{ON}$ and $\mathrm{OFF}$ duration having exponential distributions. A video flow is modelled by modified MPEG source, where there are three types of frame, i.e., $\mathrm{I}, \mathrm{B}$ and $\mathrm{P}$ frames. Each frame size is determined by a Lognormal distribution with a specified mean and standard deviation. A video source generates 24 frames per second. Data flow is modelled by Poisson arrival with truncated Pareto distributed burst size. Table 1 shows the traffic model parameters used in this simulation, where voice and video traffic are assumed to be real time flow while data traffic is assumed to be non real time flow.

\subsection{Results and Discussions}

To evaluate the performance of DGFQ, we choose throughput, average delay and maximum delay as performance measures. The definitions and simulation results are given in the following.

Throughput. We used throughput as a fairness measure, which is total transmitted packets during the whole simulation duration, say, 1000 seconds. As described in previous Section 3 basically DGFQ does rearrange the service order for the packet from a set of flows according to the individual flow characteristics. 

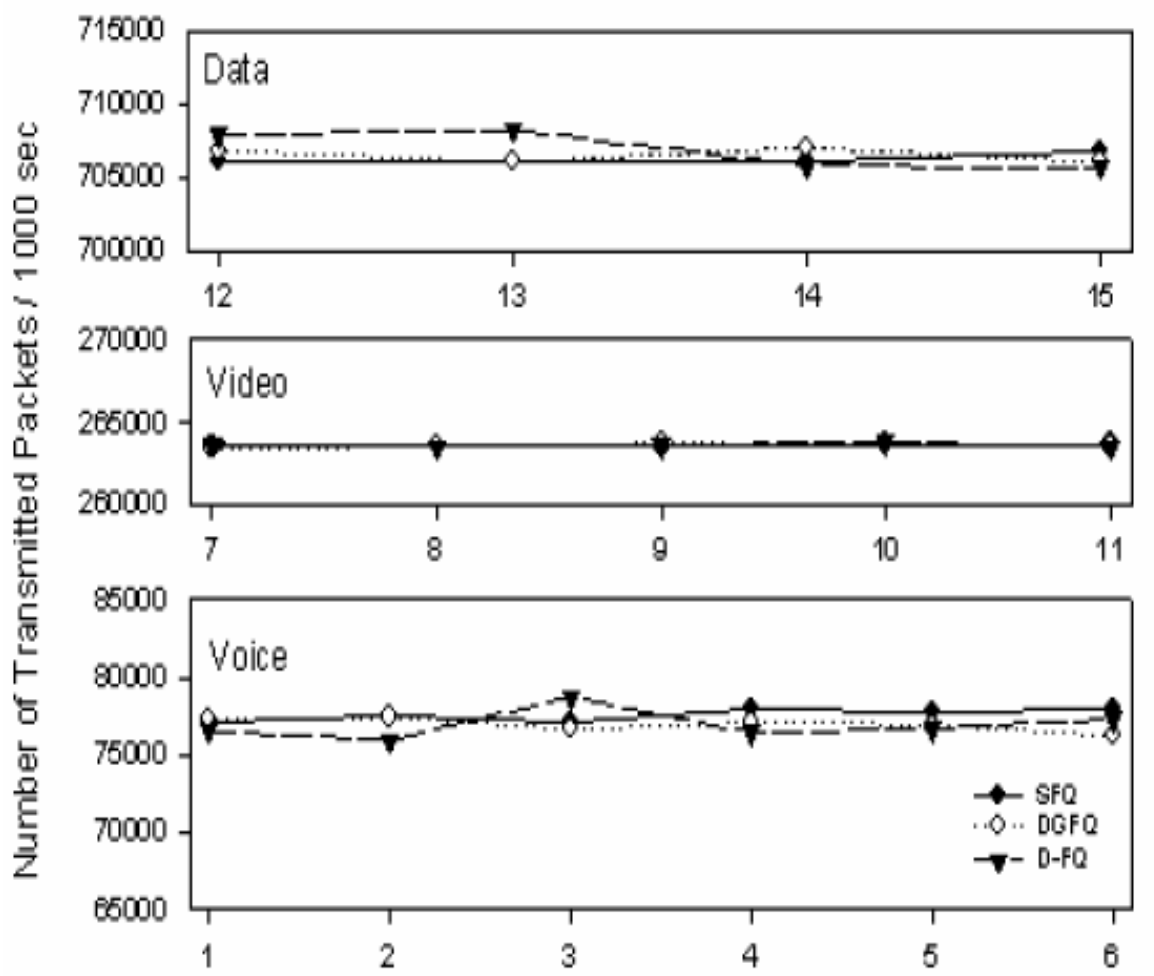

Flows

Fig. 2. Total Transmitted Packets for Each Flow by Three Schemes.

This is the only difference from SFQ. Thus conceptually, there is no changes from the results of SFQ, as far as throughput is concerned. Again, fairness among flows is guaranteed analytically by (5). Therefore, as shown in the Fig. 2, it is natural to conclude that there is no difference in throughput for respective flow classes, i.e., voice, video and data.

Average delay. In our work average delay is defined as the average time interval between the arrival and departure of a packet for a certain time duration. To compare the average delay of three schemes, we choose 3 classes of 6 realtime video flows, then assign $\alpha$ value for each class, 1.0 (for SFQ), 0.8 and 0.6 respectively.

As shown in the Fig. [3, the service differentiation coefficient $\alpha$ is the key parameter to control delay performance. When $\alpha=1$ our proposed scheme is identical to the start time fair queueing(SFQ) scheme. With varying $\alpha$ we can 


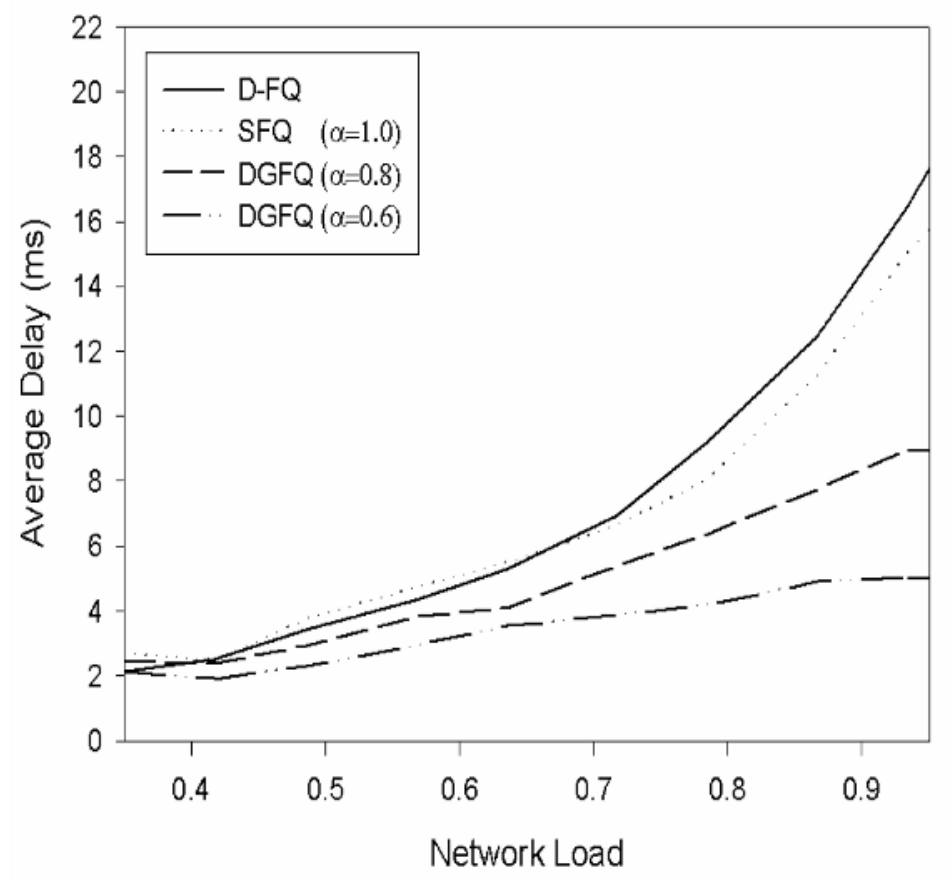

Fig. 3. Average Delay for Some Values of $\alpha$ with Varying Average Utilization.

handle classes of flows, having different delay bound requirements, maintaining the same fairness guarantees of flows. From the figure, our new scheme outperforms D-FQ and SFQ, independent of network load conditions. Specifically, with lower value of $\alpha$, we can precisely control average delay requirement. Naturally, average delay increases for all scheme with increasing network load.

Maximum delay. The maximum delay is another critical performance measure for real time multimedia flows. We define maximum delay as the maximum time interval between the arrival and departure of a packet in the system in a certain duration of time, say, simulation duration. We can get the results simultaneously with average delay from the same simulation. From the figure (Fig. 4), we can conclude that maximum delay could be also controllable with our new scheme, though the performance is not as good as for the average delay case. Specifically, as far as for the low network load conditions are concerned, say below $60 \%$ of full network load, maximum delay performance is comparable to that of D-FQ. On the contrary, for relative high load conditions, say above $80 \%$ of full network load, the maximum delay performance of DGFQ get worse with increasing network load. However, in either case, DGFQ controls the maximum delay more tightly than SFQ algorithm does. 


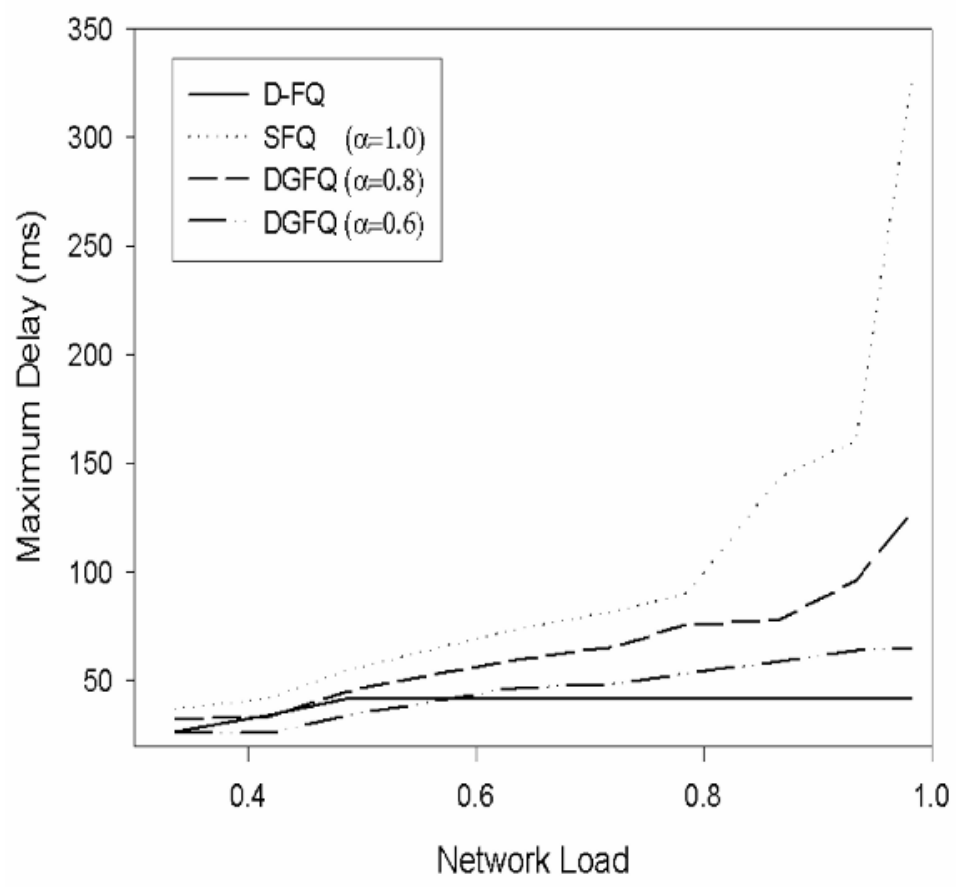

Fig. 4. Maximum Delay for Some Values of $\alpha$ with Varying Average Utilization.

For the numerical details, the target performance measure, i.e., maximum delay bound of $50 \mathrm{~ms}$, typical value for the realtime video traffic, is satisfied by DGFQ for the network load up to $60 \%$, when $\alpha=0.6$, while for SFQ, which is $\alpha=1.0$ case, meets the target only for the low load condition, say, below 30 $\%$ of full network load. D-FQ remains in the satisfied condition all the way by virtue of complex explicit delay guarantee (EDG) algorithm. It is noteworthy that our proposed scheme could manage maximum delay guarantee by simpler mechanism than D-FQ. In addition, delay performance is still under control of $\alpha$ even in the heavy load conditions where traffic congestion could be occured. As shown in the figures (Fig. 4 and Fig. 3) delay is more tightly controlled when $\alpha=0.6$ than when $\alpha=0.8$ for high load conditions.

\section{Conclusion}

We proposed a new delay guaranteed fair queueing scheme, DGFQ. This scheme provides better delay performance for DG class flows than NG class flows at the same fairness guarantee. Moreover, this scheme guarantees bounded delay of multimedia services with simpler algorithm than D-FQ [7] which explicitly guaranteeing bounded delay with rather complex queueing policy. From the simulated 
results, our proposed scheme outperforms for average delay than other schemes in comparison. Proposed scheme performs well for maximum delay bounds except for the high network load conditions with no degradation of fairness guarantees. With some modifications and performance tuning, our proposed DGFQ scheme will be a good alternatives for the fair queueing algorithm guaranteeing QoS, in the context of delay, for the multimedia wireless packet networks.

Finally, we just consider about error-free wireless channel which is too idealistic to apply our work in the practical systems. So, much more work should be done for the error-prone wireless channel case as a future work.

Acknowledgement. This work is supported in part by BK21 Project, MOE and ERC-UFON, MOST.

\section{References}

1. H. Fattah and C. Leung, "An overview of scheduling algorithms in wireless multimedia networks, "IEEE Wireless Communications, Oct. 2002, Vol.9 No.5, pp. 76-83.

2. A. K. Parekh and R. G. Gallager, "A generalized processor sharing approach to flow control in integrated services networks: the single-node case , "IEEE/ACM Transactions on Networking, Jun. 1993, Vol.1 No.3 pp. 344-357.

3. A. Demers, S. Keshav, and S. Shenker, "Analysis and simulation of a fair queueing algorithm," Proc. ACM SIGCOMM, Sep. 1989, pp. 1-12.

4. S. J. Golestani, "A self-clocked fair queueing scheme for high speed applications," Proc. INFOCOM94, Apr. 1994, pp. 636-646.

5. P. Goyal, H. M. Vin and H. Cheng, "Start-Time Fair Queueing: A Scheduling Algorithm for Integrated Services Packet Switching Networks," IEEE/ACM Transactions on Networking, Oct. 1997, Vol.5 No.5, pp. 690-704.

6. F. cuomo, A. Baiocchi and R. Cauteiier, "A MAC Protocol for a Wireless LAN Based on OFDM-CDMA," IEEE commun. Magaz., Sep. 2000, pp. 152-159.

7. S. Lee, K. Kim, A. Ahmad, "Delay and data rate decoupled fair queueing for wireless multimedia networks," Proc. GLOBECOM '02, 2002, vol. 1, pp.946-950.

8. H. Zang, "Service disciplines for guaranteed performance service in packet switched networks," Proc. of the IEEE, 1995, vol. 83, no. 10, pp. 1374-1399.

9. V. Bharghavan, A. Demers, S. Shenker, and L. Zhang, "MACAW: A Medium Access Protocol for Indoor Wireless LAN's," ACM Comput. Commun. Rev., Oct. 1995, vol. 24 , pp. $215-225$. 